\title{
HOLONOMY AND AVERAGING IN FOLIATED SETS
}

\author{
S. E. GOODMAN \& J. F. PLANTE
}

\begin{abstract}
Introduction
In the present note we formulate a general averaging condition which guarantees the existence of a holonomy invariant measure (in the sense of [7]) for a foliation of a compact set. This condition is more general than non-exponential growth and may be related to the condition "closed at infinity" introduced by D. Sullivan [10]. In $\S 2$ we show that a nonproper leaf with trivial holonomy satisfies this averaging condition if its first cohomology with compact supports is nontrivial but finitely generated. Finally, applications are made of this result using the observation that for certain foliated sets (e.g., compact minimal sets containing more than one leaf) the conditions "nonproper" and "trivial holonomy" are generic in the sense of Baire category. Since we use terminology and notation from [7], some familiarity with [7] would be helpful to the reader.
\end{abstract}

\section{Averaging sequences}

Throughout this section $X$ will be a metric space and $\Gamma$ will be a pseudogroup of homeomorphisms of $X$ generated by a fixed symmetric generating set $\Gamma_{0}$. (To say $\Gamma_{0}$ is symmetric means that $\gamma \in \Gamma_{0}$ implies $\gamma^{-1} \in \Gamma_{0}$.) We refer the reader to [7] for further definitions and background.

If $A$ is a subset of $X$, then for each $\gamma \in \Gamma$ we define the difference set $\Delta_{\gamma}(A)$ by

$$
\Delta_{\gamma}(A)=\{x \in X \mid x \in A \text { and } \gamma x \notin A\} \cup\{x \in X \mid x \notin A \text { and } \gamma x \in A\}
$$

with the convention that $\gamma x \notin A$ holds if $\gamma x$ is not defined. We denote the cardinality of a set $A$ by $|A|$. The following notion is analogous to the Følner condition for amenability in discrete groups [3].

\footnotetext{
Communicated by S. Smale, June 27,1977 . The first author was partially supported by NSF grants MCS76-05988 and MCS72-05055A04, and the second by NSF Grant MCS76-06011.
} 
Definition. An averaging sequence for $\left(X, \Gamma_{0}\right)$ is a sequence $\left\{A_{n}\right\}$ of finite subsets of $X$ such that for every $\gamma \in \Gamma_{0}$

$$
\frac{\left|\Delta_{\gamma}\left(A_{n}\right)\right|}{\left|A_{n}\right|} \rightarrow 0 \text { as } n \rightarrow \infty .
$$

If $X$ is compact and $\Gamma_{0}$ is finite, we will say that the pair $\left(X, \Gamma_{0}\right)$ is compact. The following result tells us that when $\left(X, \Gamma_{0}\right)$ is compact, the existence of an averaging sequence implies the existence of a finite $\Gamma$-invariant Borel measure on $X$. If $\left\{A_{n}\right\}$ is a sequence of subsets of $X$, the set of limit points of $\left\{A_{n}\right\}$, denoted $\lim \left\{A_{n}\right\}$, is the set of points of the form $\lim _{n \rightarrow \infty} x_{n}$ with $x_{n} \in A_{n}$.

1.1. Proposition. If $\left(X, \Gamma_{0}\right)$ is compact, and $\left\{A_{n}\right\}$ is an averaging sequence, then there is a (nontrivial) finite $\Gamma$-invariant Borel measure on $X$ with support contained in $\lim \left\{A_{n}\right\}$.

Proof. We simply construct a sequence of functionals by averaging. If $C(X)$ is the space of continuous real-valued functions on $X$, we define functionals $I_{n}: C(X) \rightarrow \mathbf{R}$ by

$$
I_{n}(f)=\frac{1}{\left|A_{n}\right|} \sum_{x \in A_{n}} f(x) .
$$

Passing to a subsequence, if necessary, the $I_{n}$ converge in the weak* topology to a nonnegative functional $I$ which determines the desired measure $\mu$ by the representation theorem of Riesz. The averaging sequence condition implies that $I$ and $\mu$ are $\Gamma$-invariant since for $\gamma \in \Gamma_{0}$ and $f \in C(X)$ such that $f \equiv 0$ outside the range of $\gamma$ we have

$$
|I(f \circ \gamma)-I(f)| \leqslant \sup _{x \in X}|f(x)| \lim _{n \rightarrow \infty} \frac{\left|\Delta_{\gamma}\left(A_{n}\right)\right|}{\left|A_{n}\right|}=0 .
$$

It is clear from this construction that $\mu(X)=1$ and $\operatorname{support}(\mu) \subset$ $\lim _{n \rightarrow \infty}\left\{A_{n}\right\}$. This proves Proposition 1.1.

The existence of an averaging sequence is a very natural condition to impose to get an invariant measure. We now formulate a related condition which has a more geometric flavor.

Definition. The $\Gamma_{0}$-boundary of a set $A \subset X$ is the set $A$ defined by

$$
\partial A=\left\{x \in A \mid \gamma x \in X-A \text { for some } \gamma \in \Gamma_{0}\right\} .
$$

1.2. Proposition. If $\left\{A_{n}\right\}$ is a sequence of finite subsets of $X$ such that

$$
\frac{\left|\partial A_{n}\right|}{\left|A_{n}\right|} \rightarrow 0 \text { as } n \rightarrow \infty,
$$

then $\left\{A_{n}\right\}$ is an averaging sequence. 
Proof. Evidently

$$
\Delta_{\gamma}\left(A_{n}\right) \subset \partial A_{n} \cup \gamma^{-1}\left(\partial A_{n}\right)
$$

so

$$
\left|\Delta_{\gamma}\left(A_{n}\right)\right| \leqslant 2\left|\partial A_{n}\right|
$$

and the result follows.

Remarks. Orbits of $\Gamma$ which have nonexponential growth in the sense of [7] determine an averaging sequence by setting $A_{n}=\Gamma^{n}(x)$ (in notation of [7]) for some fixed $x \in X$. This follows since nonexponential growth implies

$$
\frac{\left|A_{n+1}-A_{n-1}\right|}{\left|A_{n}\right|} \rightarrow 0 \text { as } n \rightarrow \infty
$$

and $\partial A_{n} \subset A_{n+1}-A_{n-1}$. Another special case of an averaging sequence occurs when $\left|\partial A_{n}\right|$ remains bounded as $n \rightarrow \infty$ but $\left|A_{n}\right| \rightarrow \infty$. This situation arises in the study of foliations which have a "vanishing cycle" in the sense of Novikov. It should be noted that averaging sequences are more general than either of these examples since none of the sets $A_{n}$ has to be contained in a single $\Gamma$-orbit. For this reason it is conceivable that the averaging sequence criterion is more general than D. Sullivan's notion [10] of "closed at infinity" for a single leaf of a foliation.

\section{Foliated sets}

The terminology in this section is the same as in [7]. We suppose $M$ is a paracompact manifold, and $\mathscr{F}$ is a continuous foliation of $M$ of arbitrary dimension and codimension. A subset $S \subset M$ is said to be $\mathcal{F}$-saturated if it a union of leaves of $\mathscr{F}$. A set such as $S$ will be called simply a foliated set. Let $\mathcal{Q}$ be a countable covering of $S$ by regular distinguished neighborhoods, and let $X$ be the space of plaques corresponding to $\mathscr{Q}$. Thus $X$ is a countable disjoint union of closed disks whose dimension equals the codimension of $\mathscr{F}$. Let $X_{S}$ be the subspace of $X$ consisting of those plaques which lie in $S$. As in [7] we construct a pseudogroup $\Gamma$ (generated by a countable symmetric generating set $\Gamma_{0}$ ) of homeomorphisms of $X_{S}$ determined by intersections of plaques. A leaf $L$ in $S$ is said to have trivial holonomy in $S$ if every element $\gamma$ of $\Gamma$ which fixes a plaque $p$ in $L$ is the identity on some neighborhood of $p$ in the domain of $\gamma$. A leaf $L \subset S$ is said to be proper in $S$ if its leaf topology agrees with its topology as a subset of $S$. Otherwise it is nonproper in $S$. We will say that a leaf $L \subset S$ contains an averaging sequence if for some covering $\mathcal{Q}$ the corresponding pair $\left(X_{S}, \Gamma_{0}\right)$ contains an averaging sequence each set of 
which contains only plaques from $L$. We say $S$ contains an averaging sequence if $\left(X_{S}, \Gamma_{0}\right)$ does.

The following result gives a condition on a leaf $L$ involving its first cohomology with compact supports which guarantees that $L$ contains an averaging sequence. We will always assume cohomology groups to have integer coefficients.

2.1. Theorem. Let $L$ be a leaf in a foliated set $S$ which is nonproper in $S$ and has trivial holonomy in $S$. If $H_{C}^{1}(L) \neq 0$ is finitely generated, then $L$ contains an averaging sequence.

Proof. Let $\mathcal{L}$ be the nerve of the covering of $L$ by plaques which correspond to interior points of $X$. Choosing the original covering $\mathscr{U}$ sufficiently fine we have $H_{C}^{1}(\mathcal{L})=H_{C}^{1}(L)$. Since $H_{C}^{1}(\mathcal{L})=\lim H^{1}(\mathcal{L}, \mathcal{L}-\mathcal{K})$ (as $\mathcal{K}$ runs over finite closed subcomplexes of $\mathcal{L}$ ) is finitely generated, we can choose a finite closed subcomplex $\mathscr{K}$ such that $H^{1}(\mathcal{L}, \mathcal{L}-\mathscr{K}) \rightarrow H_{C}^{1}(\mathcal{L})$ is surjective. Thus any element of $H_{C}^{1}(\varrho)$ is represented by a continuous map $\zeta$ : $\mathcal{L} \rightarrow S^{1}$ which has support in $\mathcal{K}$, i.e., $\mathcal{E}-\mathscr{K}$ is sent to the basepoint of $S^{1}[9$, p. 428].

We assume that $L$ contains no averaging sequence and show $H_{C}^{1}(\mathcal{L})=0$. Since $L$ is nonproper and has trivial holonomy, we can (Reeb Stability Theorem) find a sequence of disjoint isomorphic (to $\mathcal{K}$ ) complexes $\mathcal{K}_{n} \subset \mathcal{L}$ which converge to $\mathcal{K}$ in the sense that corresponding vertices (plaques) of the $\mathcal{K}_{n}$ converge in $X$ to a vertex (plaque) of $\mathcal{K}$. Let $\zeta: \mathcal{L} \rightarrow S^{1}$ have support in

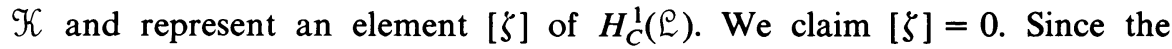
complexes $\mathcal{K}_{n}$ are isomorphic to $\mathscr{K}$ we can "lift" $\zeta$ to get a map $\zeta_{n}: \mathcal{L} \rightarrow S^{1}$ with support in $\mathcal{K}_{n}$. We will show that for $n$ sufficiently large, $\left[\zeta_{n}\right]=0$. Because of the way $\mathscr{K}$ was chosen there are maps $\eta_{n}: \mathcal{L} \rightarrow S^{1}$ with support in $\mathscr{K}$ such that $\left[\zeta_{n}\right]=\left[\eta_{n}\right]$. Letting $\xi_{n}=\zeta_{n}-\eta_{n}$ we have $\left[\xi_{n}\right]=0$ and $\operatorname{support}\left(\xi_{n}\right)$ $\subset \mathcal{K} \cup \mathcal{K}_{n}$. Thus $\xi_{n}$ lifts to a map $\tilde{\xi}_{n}: \mathcal{L} \rightarrow \mathbf{R}$ which has compact support. If $\xi_{n}$ has nonempty support, then changing the sign of $\xi_{n}$ if necessary we may assume there is a $t_{0}>0$ which is in the interior of the interval $\tilde{\xi}_{n}(\varrho)$ and does not project to the basepoint in $S^{1}$. Let $A_{n}$ be the (finite) set of vertices (plaques) $p$ such that $\tilde{\xi}_{n}(p) \geqslant t_{0}$. Note that $\partial A_{n} \subset K_{\mathcal{U}} \cup \mathcal{K}_{n}$ ( $\partial$ in the sense of the previous section). To see this let $p$ be a vertex in $\partial A_{n}$. This means there is a point $x \in \mathcal{L}$ lying in an edge of which $p$ is one of the endpoints such that $\tilde{\xi}_{n}(x)=t_{0}$. Since $t_{0}$ does not project to the basepoint in $S^{1}$ the edge (and, in particular, the endpoint $p$ ) lies in $\mathscr{K} \cup \mathcal{K}_{n}$. Now $\left|\partial A_{n}\right|$ is bounded above for all $n$ by twice the number of vertices in $\mathscr{K}$. Since we are assuming that $\left\{A_{n}\right\}$ is not an averaging sequence, this means that $\left|A_{n}\right|$ is also bounded because of (1.2). If $\left[\zeta_{n}\right] \neq 0$, the compact set $\tilde{\xi}_{n}^{-1}\left(\left[t_{0}, \infty\right)\right)$ has a connected component 
intersecting both $\mathcal{K}_{n}$ and $\mathscr{K}$. (Otherwise, redefine $\xi_{n}$ by letting $\tilde{\xi}_{n}$ be zero on connected components of the support of $\tilde{\xi}_{n}$ which do not intersect $\mathcal{K}_{n}$.) On the other hand, the distance in $\mathcal{E}$ from $\mathcal{K}_{n}$ to $\mathscr{K}$ (i.e., the minimum number of edges it takes to connect $\mathscr{K}$ and $\mathscr{K}_{n}$ ) goes to infinity as $n$ does. Hence for $n$ sufficiently large we may take $\eta_{n}$ to be trivial, i.e., $\left[\zeta_{n}\right]=0$.

By renumbering we may assume $\left[\zeta_{n}\right]=0$ for all $n$. Let $N$ be an upper bound for $\left|A_{n}\right|$, and let $\Re \subset \mathcal{E}$ be the subcomplex spanned by all vertices (plaques) which can be reached from a plaque in $\mathscr{K}$ by a plaque chain [7] of length $\leqslant N$. Again since $L$ has trivial holonomy $\Re$ lifts to a sequence of complexes $\mathscr{T}_{n} \supset \mathcal{K}_{n}$ ( $n$ sufficiently large) which converge to $\mathscr{T}$. Thus $\zeta_{n}$ can be projected back down to $\zeta$ for $n$ sufficiently large, and we conclude that $[\zeta]=0$. This completes the proof of Theorem 2.1.

2.2. Corollary. Let $S$ be a compact foliated set, and $L \subset S$ a nonproper leaf with trivial holonomy such that $H_{C}^{1}(L) \neq 0$ is finitely generated. Then there exists a nontrivial holonomy invariant measure in the sense of [7].

Proof. Corollary 2.2 follows immediately from (1.1), (2.1) and results of [7].

Remark. By lifting intersecting cycles in the same manner in which the $\mathcal{K}_{n}$ were obtained for $\mathcal{K}$ in the proof of Theorem 2.1, it can be shown that for a leaf $L$ satisfying the hypotheses of (2.1) the homomorphism $H_{C}^{1}(L) \rightarrow H^{1}(L)$ is trivial. This says that $L$ has no codimension-one handles and at least 2 ends. For example, if $L$ is an oriented surface it is homeomorphic to the plane with a finite (nonzero) number of points removed. For codimension-one foliations of 3-manifolds one can show, using Novikov's compact leaf theorem, that $L$ has at most 2 ends and, hence, must be a cylinder.

\section{Generic leaves in foliated sets}

In the previous section we considered a leaf which was nonproper and had trivial holonomy. In this section we observe that under reasonable conditions such leaves are plentiful.

Given a foliated set $S$ and a covering $\mathcal{Q}$ by distinguished neighborhoods we consider the pair $\left(X_{S}, \Gamma_{0}\right)$ as described in the previous section. A subset of $X_{S}$ is residual if it contans a countable intersection of open dense sets. According to the Baire category theorem a residual subset of $X_{S}$ must be dense in $X_{S}$ and, in particular, be nonempty. We denote by $T$ the subset of $S$ which is the union of all leaves which have trivial holonomy in $S$. The following fact [2], [4] was pointed out by us by D. Epstein. It says that trivial holonomy is a generic property for leaves of $S$. 
3.1. Proposition. $T$ is residual in $S$.

Proof. It suffices to show $X_{T}$ is residual in $X_{S}$. The set $\Gamma_{0}$ is countable, and we denote by $\Gamma^{*}$ those elements of the pseudogroup generated by $\Gamma_{0}$ which have maximal domain and are determined by a word in the elements of $\Gamma_{0}$. A plaque $p \in X_{S}$ is in $X_{T}$ if for every $\gamma \in \Gamma^{*}$ one of the following is true:

(i) $\gamma(p) \neq p$ (this includes the case $\gamma(p)$ undefined).

(ii) $\gamma(p)=p$, and $\gamma$ is the identity on a neighborhood of $p$ in the domain of $\gamma$.

For each $\gamma$, the set of $p$ satisfying (i) and (ii) is open and dense in $X_{S}$. Since the set of words in $\Gamma_{0}$ is countable we conclude that $X_{T}$ is residual in $X_{S}$.

The following result is essentially an old observation of G. D. Birkhoff. Let $D \subset S$ be the union of leaves which are dense in $S$.

3.2. Proposition. If $D$ is nonempty, then it is residual in $S$.

Proof. Let $\left\{U_{i} \mid i \in \mathbf{Z}^{+}\right\}$be a countable neighborhood base for $X_{S}$. A leaf $L$ is in $D$ if and only if $L$ contains a plaque in $U_{i}$ for every $i$. Let $S_{i}$ be the union of leaves of $S$ which contain a plaque in $U_{i}$. $S_{i}$ is an open set for each $i$. If some leaf is dense in $S$, then $S_{i}$ is also dense. Hence $D=\cap_{i} S_{i}$ is a residual set.

3.3. Corollary. If some leaf is dense in $S$, then $D \cap T$ is residual in $S$.

The foliated set $S$ is perfect if every plaque $p$ contained in $S$ is a limit of a sequence of plaques $\left\{p_{n}\right\}$ in $S$ such that $p_{n} \neq p$ for all $n$. When $S$ is perfect it is clear that any leaf which is dense in $S$ must be nonproper. If $x \in S$ we denote by $L_{x}$ the leaf through $x$.

3.4. Corollary. If $S$ is a perfect foliated set which contains a dense leaf, then for a residual (in particular nonempty) set of points $x \in S, L_{x}$ is nonproper and has trivial holonomy.

The following results follow from Theorem 2.1 and Corollary 3.4.

3.5. Theorem. Let $S$ be a perfect foliated set which contains a dense leaf but does not contain an averaging sequence. Then for a residual set of points $x \in S$, $H_{C}^{1}\left(L_{x} ; \mathbf{Z}\right)$ is either trivial or infinitely generated.

3.6. Corollary. If $S$ is a compact perfect foliated set which contains a dense leaf but does not support an invariant measure (in the sense of [7]), then for a residual set of points $x \in S, H_{C}^{1}\left(L_{x} ; \mathbf{Z}\right)$ is trivial or infinitely generated.

\section{Applications and examples}

Both extremes stated in the conclusion of Corollary 3.6 do occur. For example if $\mathscr{F}$ is the stable manifold foliation of a transitive Anosov flow [1] on a compact manifold, then every leaf is dense and all but countably many of them are homeomorphic to $\mathbf{R}^{k}$ ( $k=$ leaf dimension). The other extreme is 
illustrated, for example, by a construction of M. Hirsch [5]. In his examples the leaves all have infinitely many ends.

Let $M$ be a compact manifold, and $\mathscr{F}$ a $\bigodot^{0}$ foliation of arbitrary dimension and codimension. Since an $\mathscr{F}$-minimal set which is not a single compact leaf is an example of a perfect foliated set, Corollary 3.6 yields

4.1. Corollary. If $\Re$ is an $\mathscr{F}$-minimal set such that $H_{C}^{1}(L ; Z)$ is nontrivial and finitely generated for every leaf $L$ in $\Re$, then there is a nontrivial $\mathscr{F}$-invariant measure whose support is $\Re$.

If $\mathscr{F}$ is $\bigodot^{2}$ and has codimension one, and $\mathscr{K}$ is an exceptional minimal set, then $\mathfrak{T}$ cannot support an $\mathscr{F}$-invariant measure [6]. Thus we have the following.

4.2. Corollary. If $\mathfrak{T}$ is a minimal set of a $\complement^{2}$ codimension-one foliation, and $H_{C}^{1}(L ; \mathbf{Z})$ is nontrivial and finitely generated for every leaf $L$ in $\Re$, then either Th is a single compact leaf or $\Re=M$.

Remarks. (a) In the case $\Re=M$ of Corollary 4.2 much more can be said about the structure of $\mathcal{F}$. The manifold $M$ fibers over $S^{1}$ and the leaves of $\mathcal{F}$ are pairwise diffeomorphic. If $L$ is a leaf of $\mathcal{F}$, then $L$ has precisely two ends, and the homomorphism $H_{C}^{1}(L ; \mathbf{Z}) \rightarrow H^{1}(L ; \mathbf{Z})$ is trivial. For example, if $L$ is an oriented surface, it must be a cylinder.

(b) The special case of Corollary 4.2, when $\operatorname{dim} M=3$ and all leaves are 2-dimensional cylinders, has also been proved by G. Hector [4].

\section{References}

[1] D. Anosov, Geodesic flows on closed Riemannian manifolds with negative curvature, Proc. Steklov Inst. Math., Amer. Math. Soc., Vol. 90, 1969.

[2] D. Epstein, K. Millett \& D. Tischler, Leaves without holonomy, J. London Math. Soc. (2). 16 (1977) 548-552.

[3] F. Greenleaf, Invariant means on topological groups, Van Nostrand, New York, 1969.

[4] G. Hector, Feuilletages en cylindres, preprint.

[5] M. Hirsch, A stable analytic foliation with only exceptional minimal sets, Dynamical Systems-Warwick, Lectures in Math. Vol. 468, Springer, Berlin, 1975, 9-10.

[6] J. Plante, Measure preserving pseudogroups and a theorem of Sacksteder, Ann. Inst. Fourier (Grenoble) 25 (1975) 237-249.

[7] __ Foliations with measure preserving holonomy, Ann. of Math. 102 (1975) 327-361.

[8] G. Reeb, Sur certaines propriétés topologiques des variétées feuilletées, Actualités Sci. Indust. Hermann, Paris, 1952.

[9] E. Spanier, Algebraic topology, McGraw Hill, New York, 1965.

[10] D. Sullivan, Cycles for the dynamical study of foliated manifolds and complex manifolds, Invent. Math. 36 (1976) 225-255.

INSTITUTE FOR ADVANCED STUDY UNIVERSITY OF NORTH CAROLINA 
